\title{
Article
}

\section{A few comments and some new results on JU-algebras}

\section{Daniel A. Romano}

International Mathematical Virtual Institute 6, Kordunaška Street, 78000 Banja Luka, Bosnia and Herzegovina.; bato49@hotmail.com

Received: 24 December 2019; Accepted: 22 March 2020; Published: 5 April 2020.

Abstract: In this article, we revisit the axioms of JU-algebras previously recognizable as 'pseudo KU-algebras', which we may call as 'weak KU-algebras' and discussed the definitions of some of their substructures. We also associate this class of algebras with the classes of BE-algebras and UP-algebras. In addition, we introduce and analyze some new classes of ideals in this class of algebras.

Keywords: JU-algebras, ideal and filter in JU-algebras, closed ideal, ag-ideal, t-ideal, ( $\star$ )-ideal and associative ideal.

MSC: 03G25.

\section{Introduction}

I n 1966, Imai and Iseki [1] introduced a notion of BCK-algebras. The concept of BE-algebra as a generalization of BCK-algebra was introduced in 2006 by Kim and Kum in [2]. The concept of KU-algebras was introduced and analyzed in 2009 in [3,4]. KU-algebras are closely related to BE-algebras. Specifically, in the article [5], the authors have shown that KU-algebra is equivalent to a commutative self-distributive BE-algebra. (A BE-algebra $\mathrm{A}$ is a self-distributive if $x \cdot(y \cdot z)=(z \cdot y) \cdot(x \cdot z)$ for all $x, y, z \in A$ ). Additionally, they have shown that every KU-algebra is a BE-algebra [5]. The concept of UP-algebras as a generalization of KU-algebras was introduced by Iampan in [6]. The concept of JU-algebras, as a generalization of KU-algebras, was introduced and analyzed in [7,8].

However, this concept was introduced in [9] by Leerawat and Prabpayak under the name 'pseudo KU-algebra. In doing so, they used the PKU designation for this class of algebra. Since then, this type of generalization of KU-algebra has been in the focus of interest of the academic community (for example see $[10,11])$.

We are more inclined to refer this concept as 'weak KU-algebra' in the same way as weak BCC-algebra [12]. However, due to the tight connection of this paper to the article [8], we will use the name 'JU-algebra' in what follows.

In this article, we revisit the axioms of JU-algebras and definitions of their substructures. We also link this class of algebras with the classes BE-algebras and UP-algebras. In addition, we introduce and analyze some new classes of ideals in this class of algebra such as closed ideal, ag-ideal, t-ideal, $(\star)$-ideal and associative ideal.

\section{Preliminaries}

In this section, we take the definitions of JU-algebras, JU-subalgebras, JU-ideals and other important terminologies and some related results from literature $[7,8]$.

\subsection{Definition and some comments}

Definition 1. [8] An algebra $(A, \cdot, 1)$ of type $(2,0)$ with a binary operation "." and a fixed element 1 is said to be JU-algebras satisfying the following axioms:

(JU-1) $(\forall x, y, z \in A)((y \cdot z) \cdot((z \cdot x) \cdot(y \cdot x))=1)$,

(JU-2) $(\forall x \in A)(1 \cdot x=x)$ and

(JU-3) $(\forall x, y \in A)((x \cdot y \wedge y \cdot x=1) \Longrightarrow x=y)$.

We denote this axiom system by [JU]. 
Lemma 1. [8] In the axioms system [JU], the following formulae are valid:

(J) $(\forall x \in A)(x \cdot x=1)$,

(J12) $(\forall x, y, z \in A)(z \cdot(y \cdot x)=y \cdot(z \cdot x))$.

Comment 1. In [3], a KU-algebra is defined as a system $(A, \cdot, 0)$ by the following axioms:

(KU-1) $(\forall x, y, z \in A)((x \cdot y) \cdot((y \cdot z) \cdot(x \cdot z))=0)$,

(KU-2) $(\forall x \in A)(0 \cdot x=x)$,

(KU-3) $(\forall x \in A)(x \cdot 0=0)$ and

(KU-4) $(\forall x, y \in A)((x \cdot y=0 \wedge y \cdot x=0) \Longrightarrow x=y)$.

We denote this axiom system by [KU]. With [wKU] we denote axiomatic system [KU] without axiom (KU-3). So, $[J U] \equiv[w K U] \equiv[P K U]$.

Recall that in the axiom system [KU], the formula $\left(\mathrm{J}_{12}\right)$ is a valid formula also [13].

If in the definition of KU-algebras we write 1 instead of 0 , then we see that any KU-algebra $A$ is a JU-algebra. Therefore, the concept of JU-algebras is a generalization of the concept of KU-algebras [8].

If we followed the formation of the concept of 'weak BCC-algebras' from the 'concept of BCC-algebras', then the name 'weak KU-algebra' could also be used for a JU-algebra by analogy with the previous one.

If $A$ is a JU-algebra, let us define $\varphi: A \longrightarrow A$ as follows;

$$
(\forall x \in A)(\varphi(x)=x \cdot 1)
$$

taking the idea from [14]. According to (JU-2), the equality $\varphi(1)=1$ is valid for mapping $\varphi$.

Comment 2. The concept of UP-algebras was introduced in 2017 in article [6] as a $(A, \cdot, 0)$ system that satisfies the following axioms:

(UP-1) $(\forall x, y, z)((y \cdot z) \cdot((x \cdot y) \cdot(x \cdot z))=0)$

(UP-2) $(\forall x \in A)(0 \cdot x=x)$,

(UP-3) $(\forall x \in A)(x \cdot 0=0)$ and

(UP-4) $(\forall x, y \in A)((x \cdot y=0 \wedge y \cdot x=0) \Longrightarrow x=y)$.

We denote this axiom system by [UP]. With [wUP] we denote axiomatic system [UP] without axiom (UP-3).

We can transform the formula (JU-1) into the formula (UP-1) using valid equation $\left(\mathrm{J}_{12}\right)$ [8] and replacing the element 1 by the element 0 . However, since formula $\left(J_{12}\right)$ does not have to be a valid formula in [UP], we conclude that there is no direct connection between [JU] and [UP]. On the other hand, the system [UP] $+\left(\mathrm{J}_{12}\right)$ is equivalent to the system [KU] according to theorems in [6], so we conclude that the system [JU] is contained in the system $[w U P]+\left(J_{12}\right)$. Therefore, any UP-algebra that additionally satisfies equality $\left(\mathrm{J}_{12}\right)$ is also a JU-algebra at the same time.

Comment 3. The concept of BE-algebras is defined in [2] as a system $(A, \cdot 1)$ satisfying the following axioms:

(BE-1) $(\forall x \in A)(x \cdot x=1)$,

(BE-2) $(\forall x \in A)(x \cdot 1=1)$,

(BE-3) $(\forall x \in A)(1 \cdot x=x)$ and

(BE-4) $(\forall x, y, z \in A)(x \cdot(y \cdot z)=y \cdot(x \cdot z))$.

We denote this axiom system by [BE]. The axiomatic system generated by axioms (B-1), (BE-3) and (BE-4) is denoted by [wBE].

It is shown in [5] that every KU-algebra is a BE-algebra. Since any KU-algebra is a JU-algebra, by Comment 1 , we get that every BE-algebra is a JU-algebra.

\subsection{An order relation}

Definition 2. [8] Let $A$ be a JU-algebra. We define a relation " $\leqslant$ " in $A$ as follows:

$$
\forall x, y \in A)(y \leqslant x \Longleftrightarrow x \cdot y=1 .
$$


According to claims $\left(\mathrm{J}_{4}\right),\left(\mathrm{J}_{5}\right),\left(\mathrm{J}_{6}\right)$, and claims $\left(\mathrm{J}_{7}\right),\left(\mathrm{J}_{8}\right)$, the relation " $\leqslant$ " is a partial order in $A$ left compatible and right reverse compatible with the internal operation in $A$ [8].

Proposition 1. Let A be a JU-algebra. Then

(1) $(\forall x, y \in A)(x \cdot \varphi(y)=y \cdot \varphi(x))$;

(2) $(\forall x, y \in A)(\varphi(x) \cdot \varphi(y) \leqslant y \cdot x)$;

(3) $(\forall x, y \in A)(x \cdot(y \cdot x) \leqslant \varphi(y))$;

(4) $(\forall x, y \in A)(\varphi(x \cdot y)=\varphi(x) \cdot \varphi(y))$;

(5) $(\forall x, y \in A)(x \leqslant y \Longrightarrow \varphi(y) \leqslant \varphi(x))$.

Proof. Relation (1) is obtained directly from $\left(\mathrm{J}_{12}\right)$ where, we put $z=1$.

If we put $x=1$ and $z=x$ in (JU-1), we get $(y \cdot x) \cdot((x \cdot 1) \cdot(y \cdot 1))=1$. This means $\varphi(x) \cdot \varphi(y) \leqslant y \cdot x$ according to the Definition 2.

If we put $z=1$ in (JU-1), we get $(y \cdot 1) \cdot((1 \cdot x) \cdot(y \cdot x))=1$. Hence $\varphi(y) \cdot(x \cdot(y \cdot x))=1$. So, we have $x \cdot(y \cdot x) \leqslant \varphi(y)$.

Relation (4) is proved in [8] as formula $\left(\mathrm{J}_{14}\right)$.

Relation (5) is a direct consequence of the right inverse compatibility of order relations with an internal operation in $A$ if we choose $z=1$.

Remark 1. The relation (5) of Proposition 1 is a direct consequence of the Proposition 1(4). Indeed, if $x \leqslant y$, then $y \cdot x=1$. Thus $\varphi(y \cdot x)=1$. Hence $\varphi(y) \cdot \varphi(x)=1$ by Proposition 1(4). This means $\varphi(x) \leqslant \varphi(y)$.

\section{Some types of JU-ideals}

Definition 3. [8] A non-empty subset $J$ of a JU-algebra $A$ is called a JU-ideal of $A$ if

(J-1) $1 \in J$ and

(J-2) $(\forall x, y \in A)((x \in J \wedge x \cdot y \in J) \Longrightarrow y \in J)$.

Lemma 2. Let J be a JU-ideal of a JU-algebra A. Then

(J-3) $(\forall x, y \in A)((x \leqslant y \wedge y \in J) \Longrightarrow x \in J)$.

Proof. Let $x, y \in A$ be such that $x \leqslant y$ and $y \in J$. Then $y \cdot x=1 \in J$ and $y \in J$. Thus $x \in J$ by (J-2).

Proposition 2. Let J be a subset of a JU-algebra such that (J-1) holds. Then the condition (J-2) is equivalent to the condition;

(J-4) $(\forall x, y, z \in A)((x \cdot(y \cdot z) \in J \wedge y \in J) \Longrightarrow x \cdot z \in J)$.

Proof. (J-4) $\Longrightarrow(\mathrm{J}-2)$. If we put $x=1, y=x$ and $z=y$ in (J-4), then we get (J-2) with respect to (JU-2).

$(\mathrm{J}-2) \Longrightarrow(\mathrm{J}-4)$. Let $x, y, z \in A$ such that $x \cdots(y \cdot z) \in J$ and $y \in J$. Then $y \cdot(x \cdot z) \in J$ and $y \in J$ by $\left(\mathrm{J}_{12}\right)$. Thus $x \cdot z \in J$ by $(\mathrm{J}-2)$.

As a consequence of the Proposition 2, we can describe some of the features of the JU-ideals as follows:

Proposition 3. Let J be a JU-ideal of a JU-algebra A. Then

(6) $\forall x, y \in A)((\varphi(x) \in J \wedge y \in J) \Longrightarrow x \cdot y \in J)$ and

(7) $(\forall x, y \in A)((x \cdot \varphi(y) \in J \wedge y \in J) \Longrightarrow \varphi(x))$.

Proof. If we put $z=y$ in $(J-4)$, we get

$$
(x \cdot(y \cdot y) \in J \wedge y \in J) \Longrightarrow x \cdot y \in J .
$$

From where, we get $(J-5)$ using $\left(J_{11}\right)$ in [8]. If we put $z=1$ in $(J-4)$, we get (7).

Definition 4. Let $J$ be a JU-ideal of a JU-algebra $A$, then

(C) $J$ is a closed ideal of $A$ if $\{x \in A: \varphi(x) \in J\}=\varphi(J) \subseteq J$ holds. 
Theorem 1. An ideal J of a JU-algebra $A$ is closed if and only if it is a subalgebra of $A$.

Proof. Assume that an ideal $J$ is a subalgebra of $A$ and $x \in J$. Then, from $1 \in J$ and $x \in J$ follows $\varphi(x)=$ $x \cdot 1 \in J$ because $J$ is a subalgebra of $A$. This means $\varphi(J) \subseteq J$.

Conversely, let an ideal $J$ of $A$ is closed and let $x, y \in J$. Then $\varphi(x) \in \varphi(J) \subseteq J$ and $y \in J$. Thus $x \cdot y \in J$ by Proposition 3(6). This means that $J$ is a subalgebra of $A$.

As usual, we will write $\operatorname{Ker} \varphi=\{x \in A: \varphi(x)=1\}$. In [8], this set is labeled by $B_{A}$.

Corollary 1. Ker $\varphi$ is a closed JU-ideal of A.

Proof. Let $x, y \in A$ be such $x \in \operatorname{Ker} \varphi$ and $x \cdot y \in \operatorname{Ker} \varphi$. Then $\varphi(x)=1$ and $1=\varphi(x \cdot y)=\varphi(x) \cdot \varphi(y)=$ $1 \cdot \varphi(y)=\varphi(y)$ by Proposition 1(4) and (JU-2). Thus $y \in \operatorname{Ker} \varphi$. So, $\operatorname{Ker} \varphi$ is a JU-ideal of $A$.

Suppose $x \in \operatorname{Ker} \varphi$ and $y \in \operatorname{Ker} \varphi$. Then, from $\varphi(x)=1$ and $\varphi(y)=1$ it follows $\varphi(x \cdot y)=\varphi(x) \cdot \varphi(y)=$ $1 \cdot 1=1$ by Proposition 1(4). Thus $x \cdot y \in \operatorname{Ker} \varphi$. So, $\operatorname{Ker} \varphi$ is a subalgebra in $A$. Therefore $\operatorname{Ker} \varphi$ is a closed JU-ideal of $A$ by Theorem 1.

In what follows, we need the following lemma.

Lemma 3. Let $J$ be a JU-ideal of a JU-algebra $A$, then

(8) $(\forall x \in A)\left(x \in J \Longrightarrow \varphi^{2}(x) \in J\right)$.

Proof. Let us first show that the following holds;

(9) $(\forall x \in A)\left(\varphi^{2}(x) \leqslant x\right)$.

This inequality immediately follows from Proposition $1(2)$ if we put $y=1$. Now, from $x \in J$ and $\varphi^{2}(x) \leqslant x$ it follows $\varphi^{2}(x) \in J$ according to (J-3).

The preceding lemma is a motive for introducing the following concept.

Definition 5. Let $J$ be a JU-ideal of JU-algebra $A$, then

(AG) $J$ is ag-ideal of $A$ if $(\forall x \in A)\left(\varphi^{2}(x) \in J \Longrightarrow x \in J\right)$ holds.

Proposition 4. Let A be a JU-algebra, then

(10) $(\forall x \in A)\left(\varphi^{3}(x)=\varphi(x)\right)$.

Proof. It has already been shown that $\varphi^{2}(x) \leqslant x$ is valid. Thus $\varphi(x) \leqslant \varphi^{3}(x)$ by Proposition 1(5) and $\varphi^{3}(x)$. $\varphi(x)=1$. On the other hand, from $1=\varphi^{2}(x) \cdot \varphi^{2}(x)=\varphi^{2}(x) \cdot \varphi(\varphi(x))$, it follows $\varphi(x) \cdot \varphi^{3}(x)=1$ according to Proposition 1(1). Thus $\varphi^{3}(x)=\varphi(x)$ by (JU-3).

Corollary 2. Ker $\varphi$ is a closed ag-ideal of $A$.

Proof. It has already been shown that $\operatorname{Ker} \varphi$ is a closed JU-ideal of $A$. Let $x \in A$ be an arbitrary element such that $\varphi^{2}(x) \in \operatorname{Ker} \varphi$. Then $\varphi(x)=\varphi^{3}(x)=1$ by (9) given in proof of Lemma 3. Thus $x \in \operatorname{Ker} \varphi$.

Lemma 4. If $J$ is a JU-ideal of a JU-algebra $A$, then $\varphi^{2}(J)=\left\{x \in A: \varphi^{2}(x) \in J\right\}$ is a JU-ideal of $A$ also. The reverse also applies: If $\varphi^{2}(J)$ is a JU-ideal of JU-algerba $A$, then $J$ is a JU-ideal of $A$ also.

Proof. Since $1 \in J$, then $\varphi^{2}(1)=1 \in J$. Thus $1 \in \varphi^{2}(J)$. Suppose $x \in \varphi^{2}(J)$ and $x \cdot y \in \varphi^{2}(J)$. Then $\varphi^{2}(x) \in J$ and $\varphi^{2}(x) \cdot \varphi^{2}(y)=\varphi^{2}(x \cdot y) \in J$ with respect to Proposition $1(4)$. Thus $\varphi^{2}(y) \in J$ and $y \in \varphi^{2}(J)$. So, $\varphi^{2}(J)$ is a JU-ideal of JU-algebra $A$.

It is obvious that $1=\varphi^{2}(1) \in J$ holds because $1 \in \varphi^{2}(J)$ is valid. Let $x, y \in A$ be such $x \in J$ and $x \cdot y \in J$. Then $\varphi^{2}(x) \in \varphi^{2}(J)$ and $\varphi^{2}(x) \cdot \varphi^{y}=\varphi^{2}(x \cdot y) \in \varphi^{2}(J)$. Thus $\varphi^{2}(y) \in \varphi^{2}(J)$ by $(J-2)$ because $\varphi^{2}(J)$ is a JU-ideal of $A$. Hence $y \in J$. Therefore, $J$ is a JU-ideal of $A$.

Theorem 2. $J$ is a closed a JU-ideal of a JU-algerba A if and only if $\varphi^{2}(J)$ is a closed JU-ideal of A. 
Proof. Let $J$ be a closed a JU-ideal of a JU-algebra $A$. Let $x \in A$ be an element such that $x \in \varphi\left(\varphi^{2}(J)\right)$. Then $\varphi(x) \in \varphi^{2}(J)$ and $\left.\varphi^{2}(\varphi(x))=\varphi\left(\varphi^{2} x\right)\right) \in J$. Thus $\varphi^{2}(x) \in \varphi(J) \subseteq J$ because $J$ is a closed ideal of $A$. Hence $x \in \varphi^{2}(J)$. From this it has shown that the ideal $\varphi^{2}(J)$ satisfies Definition 4 condition (C).

Let $J$ be a JU-ideal of a JU-algebra $A$ such that $\varphi^{2}(J)$ is a closed JU-ideal of $A$. Then, we have

$$
\varphi(J)=\varphi^{3}(J)=\varphi\left(\varphi^{2}(J)\right) \subseteq \varphi^{2}(J) \subseteq J
$$

by Proposition 4(10) and since the inclusion $\varphi^{2}(J) \subseteq J$ is obviously valid. So, $J$ is a closed JU-ideal of $A$.

Before presenting the following theorem, we will introduce the concept of JU-filters in a JU-algebra.

Definition 6. A subset $F$ of a JU-algerba $A$ is a JU-filter of $A$ if the following hold:

(F-1) $1 \in F$ and

(F-2) $(\forall x, y \in A)((x \cdot y \in F \wedge y \in F) \Longrightarrow x \in F)$.

Lemma 5. If $F$ is a JU-filter of a JU-algerba $A$, then

(F-3) $(\forall x, y \in A)(x \in F \wedge x \leqslant y) \Longrightarrow y \in F)$.

Proof. Let $x, y \in A$ be such that $x \in F$ and $x \leqslant y$. Then $x \in F$ and $y \cdot x=1 \in F$. Thus $y \in F$ by (F-3).

Lemma 6. If $F$ is a JU-filter of a JU-algebra $A$, then

(11) $(\forall x \in A)(\varphi(x) \in F \Longrightarrow x \in F)$.

Proof. If we put $y=1$ in (F-2), we immediately get (11).

Remark 2. In [8], the concept of strong JU-ideal of a JU-algebra was introduced in the way we introduced the concept of JU-filters.

Theorem 3. For a JU-ideal J of a JU-algerba A the following are equivalent:

(a) $J$ is an ag-ideal of $A$;

(b) $J$ is a JU-filter of $A$;

(c) $(\forall x, y, z \in A)(((x \cdot z) \cdot(y \cdot z) \in J \wedge y \in J) \Longrightarrow x \in J)$; and

(d) $(\forall x, y \in A)(\varphi(x \cdot y) \in J \wedge y \in J) \Longrightarrow x \in J)$.

Proof. (a) $\Longrightarrow$ (b). Let $J$ be an ag-ideal of $A$ and $x \leqslant y \wedge y \in J$. Then $y \cdot x=1 \in J$ and $y \in J$. Thus $\varphi^{2}(y) \cdot \varphi^{2}(x)=\varphi^{2}(y \cdot x)=1 \in J$ and $\varphi^{2}(y) \in \varphi^{2}(J) \subseteq J$. Hence $\varphi^{2}(x) J$ because $J$ is an ideal of $A$. From here, it is follows $x \in J$ since $J$ is an ag-ideal of $A$. Therefore, subset $J$ is a JU-filter of $A$.

(b) $\Longrightarrow$ (c). From (JU-1), written in the form $(y \cdot x) \cdot((x \cdot z) \cdot(y \cdot z))=1 \in J$, and from $(x \cdot z) \cdot(y \cdot z) \in J$ we get $y \cdot x \in J$ according to (F-2). Now, from $y \cdot x \in J$ and $y \in J$ it follows $x \in J$ according to (J-2).

(c) $\Longrightarrow$ (d). If we put $z=1$ in (c), we get $\varphi(x) \cdot \varphi(y) \in J y \in J$. hence (d) with respect to (4).

(d) $\Longrightarrow$ (a). If we put $y=1$ in (d), we get (a) with respect to (J-1).

Let's introduce the following type of JU-ideals.

Definition 7. A subset $J$ of a JU-algebra $A$ is a t-ideal of $A$ if the following hold:

(J-1) $1 \in J$ and

(t) $(\forall x, y, z \in A)(((x \cdot y) \cdot z \in J \wedge y \in J) \Longrightarrow x \cdot z \in J)$.

Lemma 7. A t-ideal of a JU-algebra $A$ is a JU-ideal of $A$.

Proof. If we put $x=1, y=x$ and $z=y$ in Dentition $7(\mathrm{t})$, we get condition (J-2).

In the following proposition we give some of the characteristics of this type of JU-ideals.

Theorem 4. Let $J$ be a t-ideal of a JU-algerba $A$, then 
(e) $(\forall x, z \in A)(\varphi(x) \cdot z \in J \Longrightarrow x \cdot z \in J)$;

(f) $(\forall x, z \in A)\left(\varphi(x) \cdot z \in J \Longrightarrow \varphi^{2}(x) \cdot z \in J\right)$; and

(g) $(\forall x \in A)(x \cdot \varphi(x) \in J)$.

Proof. If we put $y=1$ in Definition $7(\mathrm{t})$, we get (e) with respect to $(\mathrm{J}-1)$.

Let $x, z \in A$ be such that $\varphi(x) \cdot z \in J$. Then

$$
\varphi\left(\varphi^{2}(x)\right) \cdot z=\varphi^{3}(x) \cdot z=\varphi(x) \cdot z \in J \Longrightarrow \varphi^{2}(x) \cdot z \in J
$$

by (e).

To prove $(\mathrm{g})$, we note that $(x \cdot 1) \cdot(x \cdot 1)=1 \in J$ and $1 \in J$ follows $x \cdot(x \cdot 1) \in J$ by Definition $7(\mathrm{t})$. So we have $x \cdot \varphi(x) \in J$.

Proposition 5. The condition (e) of Theorem 4 is equivalent to the condition ( $t$ ) of Definition 7.

Proof. Theorem 4 already proves that $(\mathrm{t}) \Longrightarrow(\mathrm{e})$, the inverse implication $(\mathrm{e}) \Longrightarrow(\mathrm{t})$ remains to be proved.

We can write (JU-1) in the form $(y \cdot z) \cdot(y \cdot x) \leqslant z \cdot x$ according to $\left(\mathrm{J}_{12}\right)$. If we put $z=1, x=y$ and $y=x$ in the previous inequality, we get $\varphi(x) \cdot(x \cdot y) \leqslant y$. From here, it follows $\varphi(x) \cdot(x \cdot y) \in J$ by $(J-3)$ and the hypothesis $y \in J$. On the other hand, if we put $z=x \cdot y, x=z$ and $y=\varphi(x)$ in (JU-1) written in the form $(y \cdot z) \cdot(y \cdot x) \leqslant z \cdot x$, then we get $(\varphi(x) \cdot(x \cdot y)) \cdot(\varphi(x) \cdot z) \leqslant(x \cdot y) \cdot z$. Thus $(\varphi(x) \cdot(x \cdot y)) \cdot(\varphi(x) \cdot z) \in J$ by $(\mathrm{J}-3)$ and the hypothesis $(x \cdot y) \cdot z \in J$.

(e).

Now, from $(\varphi(x) \cdot(x \cdot y)) \cdot(\varphi(x) \cdot z) \in J$ and $\varphi(x) \cdot(x \cdot y) \in J$ it follows $\varphi(x) \cdot z \in J$. Thus $x \cdot z \in J$ by

Proposition 6. The condition (f) of Theorem 4 is equivalent to the condition (e) of Theorem 4.

Proof. Let $x, z \in A$ be elements such that $\varphi(x) \cdot z \in J$. Then $\varphi^{2}(x) \cdot z \in J$ by (f). On the other hand, it follows $x \cdot z \leqslant \varphi^{2}(x) \cdot z$ from (9) with respect to right reverse compatibility of the operation in $A$ with the order in $A$. Now, from $x \cdot z \leqslant \varphi^{2}(x) \cdot z$ and $\varphi^{2}(x) \cdot z \in J$ it follows $x \cdot z \in J$ by (J-3). This has shown that formula (e) is a consequence of formula (f).

As Theorem 4 already shows that $(\mathrm{e}) \Longrightarrow(\mathrm{f})$, we conclude that the condition (e) is equivalent to the condition (f).

Proposition 7. The condition ( $\mathrm{g}$ ) of Theorem 4 is equivalent to the condition (e) of Theorem 4.

Proof. Assume (g). Let $x, y, z \in A$ be elements such that $\varphi(x) \cdot z \in J$. If we put $y=x, x=z$ and $z=\varphi(x)$ in (JU-1), written in the form $(z \cdot x) \cdot(t \cdot x) \leqslant y \cdot z$, we get $(\varphi(x) \cdot z) \cdot(x \cdot z) \leqslant x \cdot \varphi(x)$. From here, it follows $(\varphi(x) \cdot z) \cdot(x \cdot z) \in J$ by $(\mathrm{J}-3)$ and the hypothesis $(\mathrm{g}): \varphi(x) \cdot x \in J$. Now, from $(\varphi(x) \cdot z) \cdot(x \cdot z) \in J$ it follows $x \cdot z \in J$ by $(\mathrm{J}-2)$ and hypothesis $\varphi(x) \cdot z \in J$. We have shown by this that $(\mathrm{g}) \Longrightarrow(\mathrm{e})$.

In Theorem 4 it is shown that $(\mathrm{t}) \Longrightarrow(\mathrm{g})$. Since $(\mathrm{t}) \Longleftrightarrow(\mathrm{g})$ is a valid formula, by Proposition 5 , we conclude that the equivalence of $(\mathrm{e}) \Longleftrightarrow(\mathrm{g})$ is a valid formula, too.

The concept of p-ideals of a JU-algebra was introduced and analyzed in [8].

Definition 8. [8] A subset $J$ of a JU-algebra $A$ is called a p-ideal of $A$ if

(J-1) $1 \in J$ and

(p) $(\forall x, y, z \in A)((z \cdot x) \cdot(z \cdot y) \in J \wedge y \in J) \Longrightarrow x \in J)$.

Lemma 8. Any p-ideal J of a JU-algebra $A$ is a JU-ideal of $A$.

Proof. If we put $z=1$ in Definition $8(\mathrm{p})$, we get (JU-2).

Theorem 5. Let J be a p-ideal of a JU-algerba A. Then

(h) $(\forall y, z \in A)((z \cdot x) \cdot \varphi(z) \in J \Longrightarrow x \in J)$;

(k) $(\forall x, z \in A)(z \cdot \varphi(z \cdot x) \in J \Longrightarrow x \in J)$;

(m) $(\forall x, z \in A)((\varphi(z \cdot x) \in J \wedge z \in J) \Longrightarrow x \in J)$; 
(n) $(\forall x \in A)(\varphi(x) \in J \Longrightarrow x \in J)$.

Proof. If we put $y=1$ in Definition $8(\mathrm{p})$, we get (h).

The condition (k) is obtained from condition (h) by applying equality (1) of Proposition 1.

If we put $y=z$ in in Definition $8(\mathrm{p})$, we get $(\mathrm{m})$.

If we put $z=1$ in (m), we get (n).

In what follows, we introduce and analyze a new type of JU-ideal in JU-algebras.

Definition 9. Let $J$ be a JU-ideal of a $A$. $J$ is a $(\star)$-ideal of $A$ if

(*) $(\forall x, y \in A)(\neg(x \in J) \wedge y \in J) \Longrightarrow x \cdot y \in J)$.

Proposition 8. Let J be $a(\star)$-ideal of a JU-algebra A. Then

(q) $(\forall x \in A)(\neg(x \in J) \Longrightarrow \varphi(x) \in J)$; and

(r) $(\forall x, y \in A)((\neg(x \cdot y \in J) \wedge y \in J) \Longrightarrow x \in J)$.

Proof. The Condition (q) is obtained by putting $y=1$ in Definition $9(\star)$.

The Condition (q) can be obtained from the contraposition of Definition $9(\star)$.

Theorem 6. An ideal J of a JU-algebra $A$ is a closed ( $(x)$-ideal if and only if $\varphi(A)=\{\varphi(x): x \in A\} \subseteq J$.

Proof. Let $J$ be an JU-ideal of $A$. If $\varphi(A) \subseteq J$, then obviously $\varphi(J) \subseteq J$ holds, i.e., the ideal $J$ is closed.

Let $x, y \in A$ be arbitrary elements. Then $y \cdot(x \cdot y) \leqslant \varphi(x) \in J$ by Proposition 1(3) and hypothesis $\varphi(A) \subseteq J$. Thus $y \cdot(x \cdot y) \in J$ by Lemma 2. Hence $x \cdot y \in J$ by $(\mathrm{J}-2)$. So, $J$ is a $(\star)$-ideal of $A$.

Let $J$ be a closed $(\star)$-ideal of $A$. For $x \in A$, we have $x \in A \vee \neg(\in J)$. If $x \in J$, then $\varphi(x) \in J$ because $J$ is a closed ideal of $A$.

If $\neg(x \in J)$, then from $\neg(x \in J) \wedge 1 \in J$ it follows $\varphi(x)=x \cdot 1 \in J$ because $J$ is a $(\star)$-ideal of $A$.

Before we finish this section, let us introduce another type of JU-ideal.

Definition 10. Let $J$ be a JU-ideal of a JU-algebra $A$. $J$ is an associative JU-ideal of $A$ is

(A) $(\forall x, z \in A)(\varphi(z) \cdot x \in J \Longrightarrow x \cdot z \in J)$.

Theorem 7. An associative ideal $J$ of a JU-algebra $A$ is closed and ag-ideal of $A$.

Proof. If we put $x=1$ in Definition $10(\mathrm{~A})$, we get $\varphi(z) \cdot 1 \in J \Longrightarrow 1 \cdot z \in J$, i.e. we get $\varphi^{2}(z) \in J \Longrightarrow z \in J$. This means that $J$ is an ag-ideal of $A$.

If we put $z=1$ in Definition $10(\mathrm{~A})$, we get $\varphi(1) \cdot x \in J \Longrightarrow x \cdot 1 \in J$, i.e. we get $x \in J \Longrightarrow \varphi(x) \in J$. So, $J$ is a closed JU-ideal of $A$.

\section{Conclusion}

In $[7,8]$, the concept of JU-algebras is introduced and analyzed. However, this concept was introduced earlier in [9] under the name 'pseudo KU-algebra'. This author is more inclined to use the term 'weak KU-algebra' for this generalization of KU-algebras. In this paper, we have introduced and analyzed the concepts of a few new types of JU-ideals of a JU-algebra such as closed ideal, ag-ideal, t-ideal, $(\star)$-ideal and associative ideal. This article opens the possibility of introducing and analyzing several different types of JU filters (Definition 6) in these algebras.

Acknowledgments: The author would like to thank the College of Natural Sciences, Jimma University for funding this research work.

Conflicts of Interest: "The author declares no conflict of interest."

\section{References}

[1] Imai, Y., \& Iseki, K. (1966). On axiom systems of propositional calculi XIV. Proceedings of the Japan Academy, Series A, Mathematical Sciences, 42(1), 19-22.

[2] Kim, H. S., \& Kum, Y. H. (2007). On BE-algebras. Scientiae Mathematicae Japanicae, 66(1), 113-116. 
[3] Prabpayak, C., \& Leerawat, U. (2009). On ideals and congruences in KU-algebras. Scienctia Magna Journal, 5(1), $54-57$.

[4] Prabpayak, C., \& Leerawat, U. (2009). On isomorphisms of KU-algebras. Scientia Magna Journal, 5(3), $25-31$.

[5] Rezaei, A., Saeod, A. B., \& Borzooei, R. A. (2014). KU-algebras are equivalent to commutative self-distributive BE-algebras. Bollettino di Matematica Pura e Applicata, 7, 1-8.

[6] Iampan, A. (2017). A new branch of the logical algebra: UP-algebras. Journal of Algebra and Related Topics, 5(1): 35-54.

[7] Ali, U., Ansari, M. A., \& Rehman, M. U. (2019). Pseudo-valuations and pseudo-metric on JU-algebras. Open Journal of Mathematical Sciences, 3(1), 440-446.

[8] Ansari, M. A., Haider, A., \& Koam, A. N. (2020). On JU-algebras and p-closure ideals. International Journal of Mathematics and Computer Science, 15(1), 135-154.

[9] Leerawat, U., \& Prabpayak, C. (2015). Pseudo KU-algebras and their applications in topology. Global Journal of Pure and Applied Mathematics, 11(4), 1793-1801.

[10] Prabpayak, C. (2017). Derivations of PKU-algebras. Far East Journal of Mathematical Sciences, 102(9), $1917-1923$.

[11] Prabpayak, C., Daowsud, K., \& Leerawat, U. (2018). Some results on fuzzy PKU-algebras. International Journal of Pure and Applied Mathematics, 118(2), 221-230.

[12] Dudek, W. A., \& Thomys, J. (2012). On some generalizations of BCC-algebras. International Journal of Computer Mathematics, 89(12), 1596-1616.

[13] Mostafa, M. S., Naby, M. A. A., \& Yousef, M. M. M. (2011). Fuzzy ideals of KU-algebras. International Mathematical Forum, 6(63), 3139-3149.

[14] Dudek, W. A., \& Thomys, J. (1990). On decompositions of BCH-algebras. Mathematicae Japonicae, 35(6), $1131-1138$.

(C) 2020 by the authors; licensee PSRP, Lahore, Pakistan. This article is an open access article distributed under the terms and conditions of the Creative Commons Attribution (CC-BY) license (http://creativecommons.org/licenses/by/4.0/). 